\title{
Southern annular mode dynamics in observations and models, Part l: The influence of climatological zonal wind biases in a comprehensive GCM
}

Article

Published Version

Simpson, I. R., Hitchcock, P., Shepherd, T. G. and Scinocca, J. F. (2013) Southern annular mode dynamics in observations and models, Part I: The influence of climatological zonal wind biases in a comprehensive GCM. Journal of Climate, 26 (11). pp. 3953-3967. ISSN 1520-0442 doi:

https://doi.org/10.1175/JCLI-D-12-00348.1 Available at https://centaur.reading.ac.uk/33005/

It is advisable to refer to the publisher's version if you intend to cite from the work. See Guidance on citing.

Published version at: http://dx.doi.org/10.1175/JCLI-D-12-00348.1

To link to this article DOI: http://dx.doi.org/10.1175/JCLI-D-12-00348.1

Publisher: American Meteorological Society

All outputs in CentAUR are protected by Intellectual Property Rights law, including copyright law. Copyright and IPR is retained by the creators or other copyright holders. Terms and conditions for use of this material are defined in the End User Agreement. 


\section{www.reading.ac.uk/centaur}

\section{CentAUR}

Central Archive at the University of Reading

Reading's research outputs online 


\title{
Southern Annular Mode Dynamics in Observations and Models. Part I: The Influence of Climatological Zonal Wind Biases in a Comprehensive GCM
}

\author{
ISLA R. SIMPSON* AND PETER HitCHCOCK ${ }^{+}$ \\ Department of Physics, University of Toronto, Toronto, Ontario, Canada \\ THEODORE G. SHEPHERD \\ Department of Physics, University of Toronto, Canada, and Department of Meteorology, University of Reading, \\ Reading, United Kingdom \\ JOHN F. SCINOCCA \\ Canadian Centre for Climate Modelling and Analysis, Environment Canada, Victoria, British Columbia, Canada
}

(Manuscript received 8 June 2012, in final form 12 October 2012)

\begin{abstract}
A common bias among global climate models (GCMs) is that they exhibit tropospheric southern annular mode (SAM) variability that is much too persistent in the Southern Hemisphere (SH) summertime. This is of concern for the ability to accurately predict future SH circulation changes, so it is important that it be understood and alleviated. In this two-part study, specifically targeted experiments with the Canadian Middle Atmosphere Model (CMAM) are used to improve understanding of the enhanced summertime SAM persistence. Given the ubiquity of this bias among comprehensive GCMs, it is likely that the results will be relevant for other climate models.

Here, in Part I, the influence of climatological circulation biases on SAM variability is assessed, with a particular focus on two common biases that could enhance summertime SAM persistence: the too-late breakdown of the Antarctic stratospheric vortex and the equatorward bias in the SH tropospheric midlatitude jet. Four simulations are used to investigate the role of each of these biases in CMAM. Nudging and bias correcting procedures are used to systematically remove zonal-mean stratospheric variability and/or remove climatological zonal wind biases. The SAM time-scale bias is not alleviated by improving either the timing of the stratospheric vortex breakdown or the climatological jet structure. Even in the absence of stratospheric variability and with an improved climatological circulation, the model time scales are biased long. This points toward a bias in internal tropospheric dynamics that is not caused by the tropospheric jet structure bias. The underlying cause of this is examined in more detail in Part II of this study.
\end{abstract}

\footnotetext{
* Current affiliation: Department of Ocean and Climate Physics, Lamont Doherty Earth Observatory, Columbia University, Palisades, New York.

+ Current affiliation: Department of Applied Mathematics and Theoretical Physics, University of Cambridge, Cambridge, United Kingdom.
}

Corresponding author address: Isla R. Simpson, Department of Ocean and Climate Physics, Lamont Doherty Earth Observatory, Columbia University, P.O. Box 100, Route 9W, Palisades, NY 10964-1000.

E-mail: isla@ldeo.columbia.edu

\section{Introduction}

Global climate models are a vital tool for the prediction of future changes to our climate system and as such there is great pressure for them to simulate all the relevant components of the climate system accurately. However, there are still certain aspects of the underlying large-scale atmospheric dynamics that are not represented correctly for reasons that are not well understood. One such feature is the dynamics of the Southern Hemisphere (SH) tropospheric midlatitude jet. Both the zonal-mean climatology and the zonal-mean 
variability of the $\mathrm{SH}$ midlatitude jet are notoriously difficult to simulate correctly, and virtually all GCMs exhibit similar biases in both these respects (Fyfe and Saenko 2006; Gerber et al. 2008a, 2010; Kidston and Gerber 2010, hereafter KG2010; Swart and Fyfe 2012a,b).

The southern annular mode (SAM) is the dominant mode of variability in the $\mathrm{SH}$ extratropical circulation in both the troposphere and stratosphere (Gong and Wang 1999; Thompson and Wallace 2000). Often defined by the first empirical orthogonal function (EOF) of zonalmean geopotential or zonal wind, it describes a latitudinal shifting of the eddy-driven midlatitude westerly jet in the troposphere and a strengthening/weakening of the polar vortex in the stratosphere. In the troposphere, many different climate forcings produce an extratropical circulation response that projects strongly onto the SAM (e.g., Thompson and Solomon 2002; Seager et al. 2003; Haigh et al. 2005; Miller et al. 2006; Lorenz and Deweaver 2007; Fogt et al. 2009). The processes that govern latitudinal shifting of the eddydriven jet in the natural variability (e.g., eddy-mean flow feedbacks and dissipation of zonal wind anomalies by surface friction) are also likely to be relevant for latitudinal shifts of the jet in response to such climate forcings. Any inaccuracies in the simulation of the present-day natural SAM variability may therefore indicate a deficiency in models that will affect our ability to predict forced circulation changes. Therefore, in order to accurately simulate both natural climate variability and forced responses, the dynamics of SAM variability must be represented correctly in GCMs.

An important characteristic of tropospheric SAM variability is its temporal persistence (Gerber et al. $2008 \mathrm{~b}$ ). Typical $e$-folding time scales of SAM variability in the observed troposphere are on the order of 10 days (Baldwin et al. 2003; Gerber et al. 2008a). While models generally capture the spatial structure of SAM variability well, simulated SAM variability tends to be much too persistent, particularly in the SH summer. Gerber et al. (2008a, 2010) performed a detailed intercomparison of SAM variability in state-of-the-art GCMs. The first of these studies examined the coupled atmosphere-ocean models of phase 3 of the Coupled Model Intercomparison Project (CMIP3) and the second focused on the coupled chemistry climate models of the Chemistry Climate Model Validation Activity, phase 2 (CCMVal-2). Both these groups of models, while coupling to different components of the climate system, were found to exhibit the same signed bias relative to reanalysis data: SAM variability was much too persistent in the $\mathrm{SH}$ summer season. In Fig. 11 of Gerber et al. (2010) it can be seen that almost all of the chemistry climate models have SAM time scales that are of the order of 2-3 times longer than those of the observed atmosphere in the summer season.

The bias in SAM time scales is of concern for our ability to accurately simulate and predict changes in SH midlatitude circulation. The fluctuation-dissipation theorem (Leith 1975) provides theoretical arguments that relate the magnitude of a forced response to the time scale of natural unforced variability. It predicts that a mode of variability that is characterized by a longer time scale will exhibit a larger magnitude of response to a forcing (that projects onto the mode) than one that is characterized by a shorter time scale. A physical explanation for this in the context of the SAM is that the feedback or dissipative processes that act to maintain or dissipate SAM anomalies in the natural variability will also likely play a role in the SAM-like response to a climate forcing. Therefore, a positive bias in SAM time scales may imply a bias in feedbacks or dissipation of SAM anomalies, which may in turn also result in the simulated SAM-like response to a forcing being too large. The SH circulation has seen a great deal of change in recent decades with ozone depletion resulting in a SAM-like poleward shifting of the tropospheric jet in the summer season (e.g., Thompson and Solomon 2002; Fogt et al. 2009). In the future, ozone recovery and anthropogenic greenhouse gas emissions are also predicted to alter SH midlatitude circulation (McLandress et al. 2011; Polvani et al. 2011). If the SAM time-scale bias does indeed reflect a problem in the dynamics of feedback or dissipative processes acting on tropospheric SAM anomalies, then we cannot be confident that GCMs have been able to simulate recent SH climate change for the right reasons or that they will accurately predict future $\mathrm{SH}$ climate change. Aside from the implications for regional-scale climate in the midlatitudes, this is also of concern for global-scale climate since the position of the midlatitude westerlies in the $\mathrm{SH}$ could influence the uptake of $\mathrm{CO}_{2}$ by the Southern Ocean (Russell et al. 2006; Swart and Fyfe 2012b) or Antarctic sea ice extent (Sigmond and Fyfe 2010).

However, it is also possible that the bias in tropospheric SAM persistence arises from the influence of variability on intraseasonal time scales of "external" forcings on the tropospheric jet. Such external forcings would not directly influence the internal feedback and dissipative processes that may dictate the response to the more secular forcings responsible for $\mathrm{SH}$ climate change. For example, Baldwin et al. (2003) demonstrated a relationship between stratospheric variability 
and tropospheric annular mode time scales. Specifically, in active stratospheric seasons, the persistence of the annular modes in each hemisphere is increased. Keeley et al. (2009) then interpreted this as the stratosphere providing an external forcing on the troposphere on intraseasonal time scales that varies interannually. So, rather than there being a change in the dynamics of SAM variability in stratospherically active seasons, stratospheric variability provides a forcing on the tropospheric jet for an extended period of time. It is possible that a bias in SAM time scales could thus arise from a bias in stratospheric variability; if so, this may pose less of a concern for the fidelity of climate responses simulated by GCMs.

Indeed, a common bias among stratosphere-resolving models is that the Antarctic polar vortex breaks down too late (Butchart et al. 2011), causing the maximum in stratospheric variability to occur too late into the summer season. This could act to enhance the summertime SAM persistence in the models. However, in a recent study with the Canadian Middle Atmosphere Model (CMAM), stratospheric variability was eliminated by nudging the zonal mean in the stratosphere toward the model climatology (Simpson et al. 2011). It was found that the zonal-mean stratospheric variability does have a significant influence on the SAM time scales but, even in the absence of zonal-mean stratospheric variability, the SAM time scales in the model are biased long compared to observations. All other boundary conditions were held fixed in these simulations, which therefore pointed to a contribution from internal tropospheric dynamics to the SAM time-scale bias. While that study demonstrated a role for internal tropospheric dynamics in contributing to the SAM time-scale bias, the experiments performed could not rule out the possibility that there is also a role for biases in stratospheric variability associated with the too-late breakdown of the vortex. This study follows on from Simpson et al. (2011) and aims to both assess the role of biases in stratospheric variability on the tropospheric SAM time-scale bias and improve our understanding of the underlying cause of the bias in internal tropospheric dynamics already found in CMAM.

The dynamics of tropospheric annular mode variability are governed by positive feedbacks between tropospheric eddies and zonal-mean flow anomalies (Robinson 1996, 2000; Lorenz and Hartmann 2001, 2003; Kidston et al. 2010). It was Lorenz and Hartmann (2001) who first demonstrated that, in the observed atmosphere, the persistence of the SAM is predominantly governed by this positive feedback by eddy momentum fluxes and the dissipation of zonal wind anomalies by surface friction. Thus, a bias in internal tropospheric dynamics leading to enhanced SAM persistence is likely to involve one of these aspects.

One of the favored candidates for giving rise to the enhanced SAM persistence in the models is a bias in climatological tropospheric jet structure (KG2010). As mentioned previously, GCMs also have difficulty simulating the climatology of the SH jet stream with it being situated too far equatorward in virtually all GCMs (Fyfe and Saenko 2006; KG2010; Swart and Fyfe 2012a,b). KG2010 examined the annual-mean SAM time scales in the CMIP3 models and found a relationship between the climatological jet latitude in the models and the time scale of SAM variability. The lower the latitude of the jet (i.e., the greater the bias in the zonal-mean climatology), the longer the SAM time scale. They also found that the models with the greater bias in jet position and SAM time scales exhibited the largest climate change response. A similar result was found by Son et al. (2010) for the SH circulation response to Antarctic ozone depletion in the CCMVal-2 models. A possible explanation of these results is that the strength of eddy feedbacks on the SAM depends on climatological jet structure. Indeed, this has been found to be the case in simplified GCMs (Son and Lee 2005; Gerber and Vallis 2007; Barnes et al. 2010; Simpson et al. 2010), and various mechanisms have been proposed to explain such a dependence based on the influence of jet structure on eddy-mean flow feedbacks (Son et al. 2008; Barnes et al. 2010; Simpson et al. 2012). It is possible that this bias in the position of the tropospheric jet results in eddy feedbacks onto the SAM that are too strong, leading to variability that is too persistent.

Given that two possible contributors to the SAM time-scale bias are 1) the bias in climatological tropospheric jet structure and 2) the bias in the timing of the stratospheric vortex breakdown, we now perform model experiments to examine the impact of both of these biases on SAM persistence. Here, in Part I of this study, we aim to determine whether improving the climatological jet structure and timing of the vortex breakdown brings the model time scales toward those of the observed atmosphere. It will be demonstrated that this is not the case. The SAM time-scale bias remains even with an improved climatological jet structure and timing of the vortex breakdown. Simpson et al. (2013, hereafter Part II) therefore proceed to examine the dynamics of eddy feedbacks on the SAM in the model to investigate whether the model differs from the observed atmosphere in this regard.

Section 2 will first outline the suite of model experiments and data to be analyzed in both parts of this study. The diagnostics used to examine the SAM persistence 
TABLE 1. Summary of CMAM experiments.

\begin{tabular}{|c|c|c|c|c|}
\hline Name & Length & Troposphere & Stratosphere & SSTs \\
\hline FREE & $100 \mathrm{yr}$ & free running & free running & obs climatology \\
\hline NUDG & $100 \mathrm{yr}$ & free running & $\begin{array}{l}\text { nudged: } k=0 \text { toward seasonally } \\
\text { varying climatology of FREE; } \\
\tau=6 \mathrm{~h} ; K \text { shown in Fig. } 1 \mathrm{a}\end{array}$ & obs climatology \\
\hline $\mathrm{BC}$ & $2 \times 39 \mathrm{yr}$ & $\begin{array}{l}\text { bias corrected: } n<21 \text {; } K \text { shown in } \\
\text { Fig. } 1 \mathrm{~b} ; \tau=24 \mathrm{~h} \text {; tendencies from } \\
\text { BC_REF }\end{array}$ & bias corrected as troposphere & obs varying \\
\hline BCNUDG & $100 \mathrm{yr}$ & $\begin{array}{l}\text { bias corrected: } n<21 ; K \text { shown in } \\
\text { Fig. } 1 \mathrm{c} ; \tau=24 \mathrm{~h} \text {; tendencies from } \\
\text { BCNUDG_REF }\end{array}$ & $\begin{array}{l}\text { nudged: } k=0 \text { toward the seasonally } \\
\text { varying ERA-Interim climatology; } \\
\tau=6 \mathrm{~h} ; K \text { shown in Fig. 1c }\end{array}$ & obs climatology \\
\hline
\end{tabular}

will then be described in section 3 , and in section 4 it will be demonstrated that the perturbations applied to the model are working as expected. Results are presented in section 5 where the influence of climatological biases in tropospheric jet structure and timing of the vortex breakdown on the SAM time scales is assessed. Discussion and conclusions are then given in section 6 .

\section{Model experiments and data}

The CMAM is used (Scinocca et al. 2008). This is a comprehensive, stratosphere-resolving GCM with T63 horizontal resolution and 71 levels in the vertical stretching from the surface to $0.0006 \mathrm{hPa}(\sim 100 \mathrm{~km})$. The model version used here is run with prescribed monthly-mean sea surface temperatures (SSTs), without interactive chemistry, and it does not exhibit a quasibiennial oscillation (QBO). Time slice, repeated annual cycle simulations are run with greenhouse gas concentrations held fixed at 1990s values.

The four experiments to be analyzed are summarized in Table 1. The first of these, a 100-yr control simulation, denoted FREE, is where the model is allowed to run freely with repeated annual cycles of boundary forcings (e.g., SSTs, sea ice, etc.). The other three simulations employ nudging, bias correcting, or both. In the nudging process, the model vorticity, divergence, and temperature are nudged toward a reference state via a simple relaxation in spectral space $-K(p)\left(X-X_{o}\right) / \tau_{N}$, where $X$ is the instantaneous value of a given field, $X_{o}$ is the reference state field, $\tau_{N}$ is the nudging time scale, and $K(p)$ is a nudging coefficient that allows for a vertical variation in the nudging strength. Since the nudging is done in spectral space, it can be chosen which zonal or total horizontal wavenumbers the nudging acts upon. The bias correction mode is similar to the nudged mode, but a repeated seasonal cycle of tendencies (rather than a relaxation) are applied to the vorticity, divergence, and temperature fields of the form $-K(p) X_{\text {tend }}$, where $X_{\text {tend }}$ is the desired tendency, as described in Kharin and Scinocca (2012). The aim of this is to alter the climatology but still allow variability to occur. In the bias corrected experiments performed here, the tendency $X_{\text {tend }}$ is designed to bring the model climatology toward the observed climatology (see below).

In the experiment NUDG, the zonal-mean (zonal wavenumber $k=0$ ) vorticity, divergence, and temperature in the stratosphere are nudged toward the seasonally varying climatology of the FREE simulation (retaining only up to the fourth harmonic of the annual cycle). The nudging coefficient increases linearly from 0 at $64 \mathrm{hPa}$ to 1 at $28 \mathrm{hPa}$ and above, as shown in Fig. 1a. Since only the zonal mean is nudged, waves can propagate freely into the stratosphere, but their influence on the zonal mean is limited by the nudging. In this

(a) NUDG

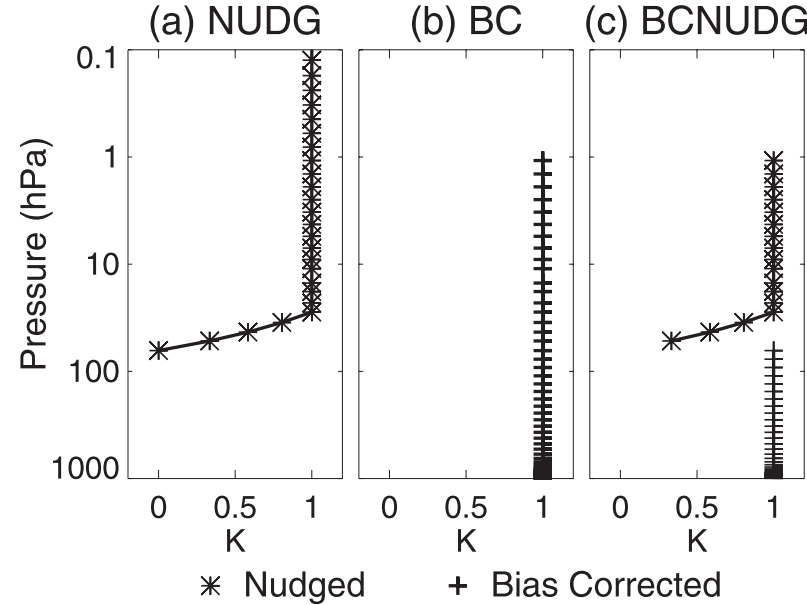

FIG. 1. Nudging and bias correcting coefficient $(K)$ as a function of vertical level for the experiments (a) NUDG, (b) BC, and (c) BCNUDG. Where no points are plotted, the model is running freely. 
TABLE 2. Reference experiments to obtain bias correction tendencies.

\begin{tabular}{|c|c|c|c|c|}
\hline Name & Length & Troposphere & Stratosphere & SSTs \\
\hline BC_REF & $40 \mathrm{yr}$ & $\begin{array}{l}\text { nudged: } n<21 \text { toward time-varying } \\
\text { ECMWF reanalysis, } \tau=24 \mathrm{~h}, \\
K(p) \text { shown in Fig. } 1 \mathrm{~b}\end{array}$ & as troposphere & obs varying \\
\hline BCNUDG_REF & $40 \mathrm{yr}$ & $\begin{array}{l}\text { nudged: } n<21 \text { toward time-varying } \\
\text { ECMWF reanalysis, } \tau=24 \mathrm{~h}, \\
K(p) \text { shown in Fig. } 1 \mathrm{c}\end{array}$ & $\begin{array}{l}\text { nudged: } k=0 \text { toward seasonally varying } \\
\text { ERA-Interim climatology, } \tau=6 \mathrm{~h}, \\
K(p) \text { shown in Fig. 1c }\end{array}$ & obs climatology \\
\hline
\end{tabular}

experiment, the nudging does not apply a net zonalmean torque in the time average, and thus the climatology remains the same as FREE. The purpose of this experiment is to obtain a simulation in which the model climatology is the same as FREE but zonal-mean stratospheric variability is absent. A comparison between this NUDG simulation and FREE was used in Simpson et al. (2011) to demonstrate that the modeled SAM time scale is biased long even in the absence of stratospheric variability.

Two additional experiments are now included, BC and BCNUDG, which employ bias correction. Before a bias corrected simulation can be performed, the required tendencies must be obtained. For BC and BCNUDG, the tendencies were obtained from two different reference simulations, BC_REF and BCNUDG_REF, which are summarized in Table 2. In BC_REF, the threedimensional (3D) vorticity, divergence, and temperature at horizontal scales down to total spherical harmonic wavenumber $n=21$ are nudged toward annually varying 6 hourly European Centre for Medium-Range Weather Forecasts (ECMWF) reanalysis data (Uppala et al. 2005; Dee et al. 2011) ${ }^{1}$ on a time scale of $24 \mathrm{~h}$ at all levels from the surface to $1 \mathrm{hPa}$, the highest level at which the ECMWF reanalysis is available. These parameters were chosen following Kharin and Scinocca (2012), who found that they resulted in instantaneous differences between CMAM and ECMWF reanalysis that were comparable to the typical magnitude of differences between different reanalysis datasets. This simulation was run from 1970 to 2009 with annually varying, monthly-mean SSTs at the lower boundary from the Hadley Centre Sea Ice and Sea

\footnotetext{
${ }^{1}$ For the input to the nudging experiments, a combination of the 40-yr ECMWF Re-Analysis (ERA-40; Uppala et al. 2005) and ERA-Interim (Dee et al. 2011) data were used. ERA-Interim was used from 1989 onward as the experiments were run before that dataset extended back to 1979. ERA-40 is used before 1989. This ERA-Interim-ERA-40 combination will be referred to as ECMWF reanalysis. The time period from 1979 to 2009 is used to obtain the bias correction tendencies, but when nudging toward ECMWF it is actually only the ERA-Interim climatology (1989-2010) that was used as the climatological spectral input from that period had been used in the Simpson et al. (2011) study.
}

Surface Temperature dataset (HadISST; Rayner et al. 2003). ${ }^{2}$ The bias correction $X_{\text {tend }}$ is defined as the average annual cycle of nudging tendencies that is derived from the 40-yr simulation, which is then smoothed by retaining only the first three harmonics of the seasonal cycle. This procedure results in a stationary (but seasonally varying) representation of the relaxational forcing employed in the nudged reference runs that is then used to remove biases in the free-running GCM. As such, $X_{\text {tend }}$ is used as a stationary forcing in the BC and BCNUDG simulations to help control the development of climatological biases in the absence of relaxational nudging. $\mathrm{BC}$ is performed with the same boundary conditions as BC_REF and the climatological, seasonally varying tendencies obtained from BC_REF applied at horizontal scales down to $n=$ 21 at each vertical level, as shown by the profile in Fig. $1 b$. The purpose of $\mathrm{BC}$ is to have a simulation where variability in both the troposphere and stratosphere can occur freely, but the climatological state at scales down to $n=$ 21 is constrained to be closer to the observed climatology than FREE. Bias correction has to be used for this purpose rather than nudging since, while nudging would improve the climatological state, it would no longer permit any variability to occur. There is some minor sensitivity of the resulting improvements from the bias correction to the parameters ( $\tau$ and horizontal scale) chosen for the reference simulation, and it is possible that another choice would result in the optimum bias correction. However, it will be shown in the following that the bias correction tendencies obtained, using the above, alleviate the climatological biases sufficiently well for the purpose of our study. Note that, since the bias correction is applied down to $n=21$, it improves the full threedimensional structure of the circulation, not just the zonal mean.

\footnotetext{
${ }^{2}$ Note that BC differs from the other simulations in that annually varying SSTs are prescribed at the lower boundary and, instead of a 100-yr time slice simulation, two realizations from 1970 to 2009 are used but with greenhouse gas concentrations held fixed. The reason for this discrepancy (and for retaining a slightly different number of harmonics for the seasonal cycle) is that BC was originally run for a different purpose (M. Sigmond 2011, personal communication)
} 
The final experiment, BCNUDG, employs bias correction in the troposphere and nudging in the stratosphere with climatological seasonally varying SSTs at the lower boundary. The tropospheric tendencies used for the bias correction were obtained from the simulation BCNUDG_REF, which ran from 1970 to 2009, with observed seasonally varying climatological SSTs and the model being nudged at all tropospheric levels up to $64 \mathrm{hPa}$ and all scales down to $n=21$, toward annually varying ECMWF reanalysis. Above $64 \mathrm{hPa}$, the zonal mean in the stratosphere in both the reference simulation and BCNUDG itself is nudged toward the ECMWF Interim Re-Analysis (ERA-Interim) climatology (climatological average from 1989 to 2010) following the profile in Fig. 1c. The purpose of this experiment is to have a simulation in which stratospheric zonal-mean variability is damped and the troposphere is allowed to vary, just like in NUDG, but in which the timing of the vortex breakdown is corrected and the climatology is closer to that of the observations.

In summary, four experiments are used. FREE is a free-running control simulation, and NUDG has the same zonal-mean climatology as FREE but zonal-mean stratospheric variability is damped by nudging. Both these simulations have the biases in the timing of the vortex breakdown and the tropospheric climatology that are common to GCMs. BC and BCNUDG are equivalent to FREE and NUDG, but the climatological biases (of the three-dimensional time mean circulation) have been improved by bias correcting in both the troposphere and stratosphere in the case of $\mathrm{BC}$ and bias correcting in the troposphere and nudging the zonal mean toward ERA-Interim in the stratosphere in the case of BCNUDG. Analysis of these four experiments can help disentangle the influence of the timing of the stratospheric vortex breakdown, as well as the biases in tropospheric climatology, on SAM persistence.

These model experiments will be compared with ERA-Interim data from 1979 to 2010 (Dee et al. 2011). ERA-Interim was obtained at 18 vertical levels from the surface to $10 \mathrm{hPa}$ and was first interpolated onto the same horizontal and vertical grid as the CMAM data using cubic splines.

\section{Time-scale diagnostic}

The SAM is defined as the first EOF of daily zonalmean geopotential height at each vertical level (Baldwin and Thompson 2009) and the primary diagnostic used is the decorrelation time scale $\tau$ of the principal component time series of that first EOF. Following the procedure of Gerber et al. (2010), for each day and each pressure level the global mean $\Phi$ is subtracted from $\Phi$ at each latitude and the resulting geopotential anomaly is deseasonalized by subtracting the climatological mean for each day of the year (and detrended for ERA-Interim), giving the input $\Phi^{\prime}$ for the EOF calculation. The first EOF of $\Phi^{\prime}$ and the corresponding principal component time series or SAM index $\mathrm{PC}(t)$, which has unit variance, is calculated on each level, using data from $90^{\circ} \mathrm{S}$ to the equator, applying a $\sqrt{\cos (\text { latitude) }}$ weighting to account for the decrease in area toward the pole (Baldwin et al. 2009).

The autocorrelation function (ACF) of PC is calculated as a function of day of the year $d$ and lag $l$ on each pressure level by

$$
\operatorname{ACF}(d, l)=\frac{\sum_{y=1}^{N_{y}-1} \operatorname{PC}(d, y) \operatorname{PC}(d+l, y)}{\sqrt{\sum_{y=1}^{N_{y}-1} \operatorname{PC}(d, y)^{2} \sum_{y=1}^{N_{y}-1} \operatorname{PC}(d+l, y)^{2}}},
$$

where $y$ is the year and $N_{y}$ is the number of years of data. This is smoothed over a 181-day window using a Gaussian filter with a full width at half maximum of around 42 days (standard deviation $\sigma=18$ days). The $e$-folding time scale $(\tau)$ of this smoothed ACF is then calculated using a least squares fit to an exponential up to a lag of 50 days.

\section{Evaluation of the nudging/bias correcting process}

Before discussing the results, it will be briefly demonstrated that the bias correction and nudging are having the desired effect.

\section{a. The influence on the climatology}

Figure 2 examines the timing of the transition from westerlies to easterlies in the Antarctic stratosphere: that is, a measure of the timing of the vortex breakdown. Figure 2a shows the timing of the transition for the climatological zonal wind for each experiment and ERAInterim. This same color scheme will be used throughout the paper: black for ERA-Interim, red for the simulations that have stratospheric variability, blue for those that do not, and dashed lines for the simulations where bias correction is applied. The main point to note is that, in FREE and NUDG, the vortex breaks down too late and in the lower stratosphere the winds do not typically transition to easterlies. However, when bias correction is applied (in $\mathrm{BC})$ or the zonal mean in the stratosphere is nudged toward ERA-Interim (in BCNUDG), the timing of the vortex breakdown is much improved and the region over which the westerly to easterly transition occurs extends lower down in the stratosphere, much like the reanalysis. 
(a) Climatology

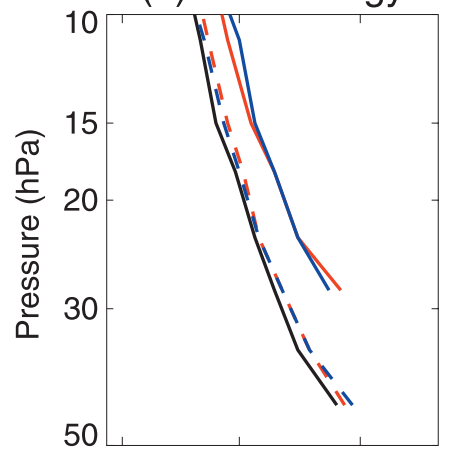

(c) FREE

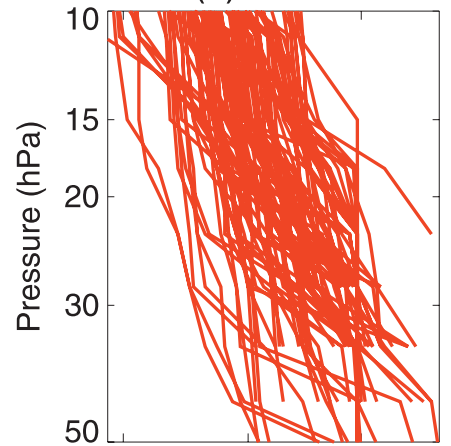

(e) BC

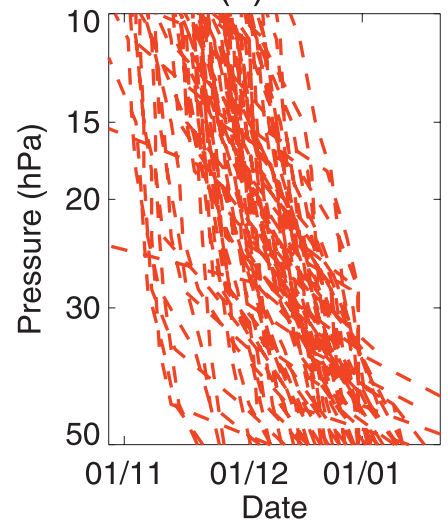

(b) ERA

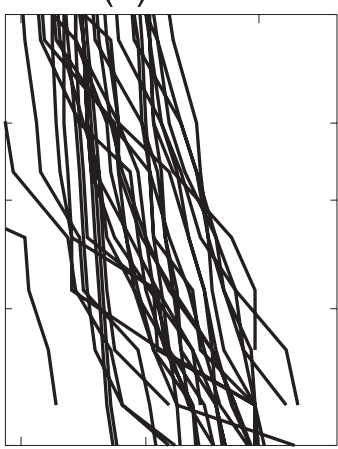

(d) NUDG

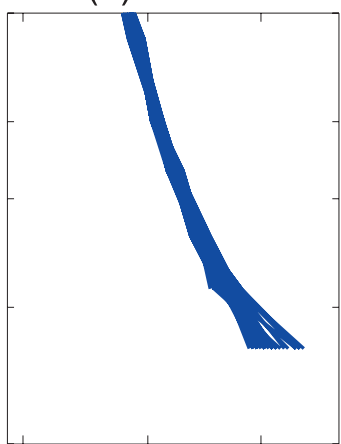

(f) BCNUDG

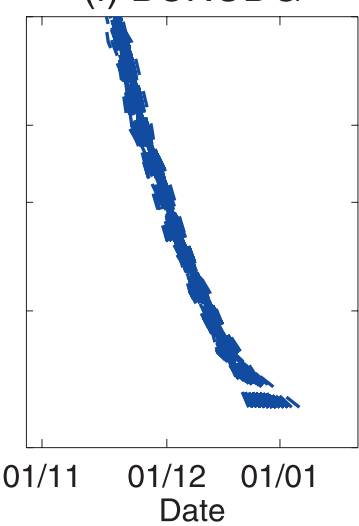

FIG. 2. Timing of the transition from zonal-mean westerlies to easterlies in the Antarctic stratosphere averaged from $50^{\circ}$ to $70^{\circ} \mathrm{S}$ [weighted by cos(latitude)] using (a) the climatological zonal-mean zonal wind for each experiment and the reanalysis, (b) individual years of the reanalysis, (c) individual years of FREE, (d) individual years of NUDG, (e) individual years of BC, and (f) individual years of BCNUDG. The color scheme in (a) is the same as that used in (b)-(f).

A second point that is demonstrated by Fig. 2 is the influence of the nudging on stratospheric variability. In ERA-Interim, FREE, and BC, where stratospheric variability is present, there is a wide range of vortex breakdown dates. This variability in the timing of the vortex breakdown corresponds to interannual variations in lower-stratospheric temperatures and winds, which

can force persistent tropospheric SAM anomalies in this season (Baldwin et al. 2003; Gerber et al. 2010; Simpson et al. 2011). There are various different mechanisms by which these perturbations in lower-stratospheric temperature and zonal wind can influence the position of the tropospheric jet, most of which involve an influence of the stratospheric perturbation on tropospheric eddies and their momentum fluxes. It is clear from Figs. 2d,f that the nudging removes this variability in the timing of the vortex breakdown so it is effective at damping stratospheric variability, as was also demonstrated in Simpson et al. (2011).

Turning now to the biases in tropospheric climatology, Fig. 3a examines the zonal-mean zonal wind on the 200-hPa level averaged over the December-February (DJF) season. This will be the main season of interest for examining the SAM time-scale bias. Comparison of the FREE and NUDG simulations demonstrates that stratospheric nudging does not impact substantially on the tropospheric climatology. The FREE and NUDG simulations also both exhibit the bias that is common among GCMs: namely, that the midlatitude zonal wind maximum is located equatorward of the reanalysis maximum. However, this equatorward bias is quite small compared to some other models (KG2010). In CMAM, the zonal winds are also too strong. These biases extend throughout the depth of the troposphere, as seen in Fig. 3b and the bias correction substantially improves the zonal wind climatology throughout the troposphere (Fig. 3c).

A demonstration of the seasonal variation of this tropospheric zonal-mean zonal wind bias and the influence of the bias correction can be seen in Figs. 3d,e. Figure $3 \mathrm{~d}$ shows the seasonal variation in zonal-mean zonal wind difference on the 200-hPa level between FREE and ERA-Interim. The positive wind bias is evident throughout the year, but it maximizes in JanuaryMarch (JFM). In Fig. 3e, the difference between BC and ERA-Interim shows that the bias correction is working effectively throughout the year. If anything, it causes the model to have a bias in the opposite direction: that is, too weak a jet. However, this bias is much smaller than that in FREE and, for our purposes of examining the effect of the climatological tropospheric biases in CMAM on the SAM time scales, the bias correction process has achieved its purpose.

Aside from biases in the zonal-mean zonal wind, biases in the three-dimensional structure of the time mean zonal wind could influence SAM persistence (Barnes and Hartmann 2010). Since the bias correction is being applied at scales down to $n=21$, it will also improve zonally asymmetric aspects of the climatological circulation. This is assessed in Fig. 4, which shows the 
(a) u, 200hPa, DJF

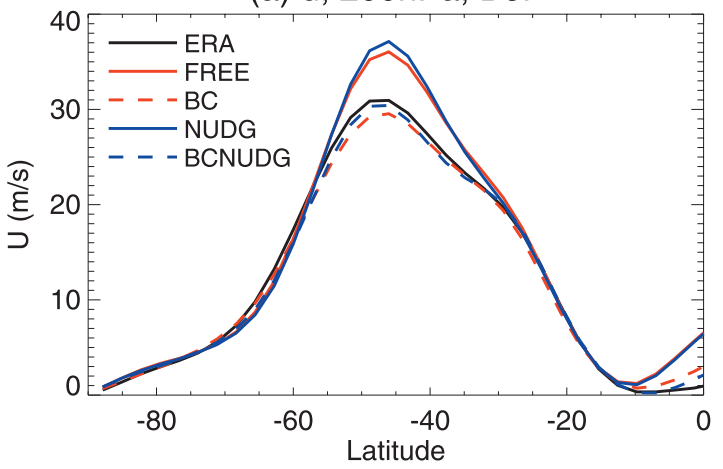

$\begin{array}{ll}\text { (b) u,FREE-ERA,DJF } & \text { (c) u,BC-ERA,DJF }\end{array}$

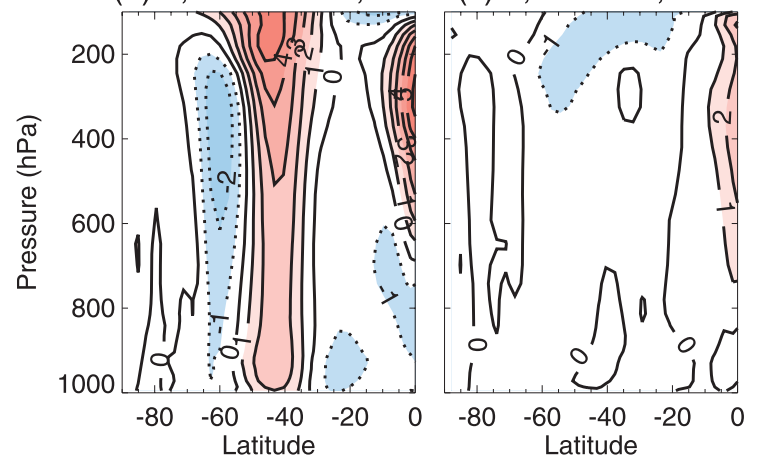

(d) u, FREE-ERA, 200hPa

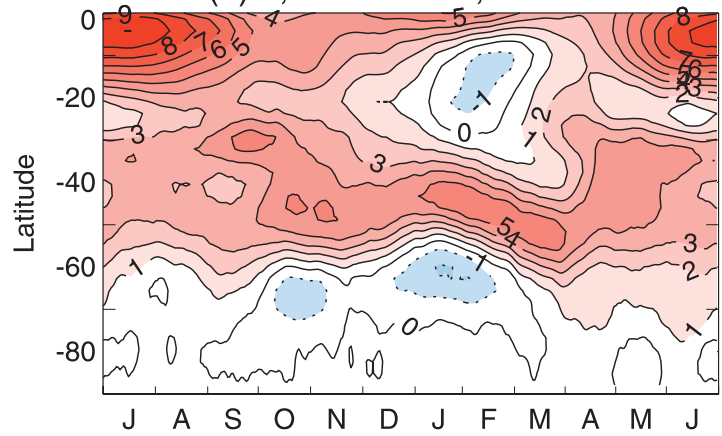

(e) u, BC-ERA, 200hPa

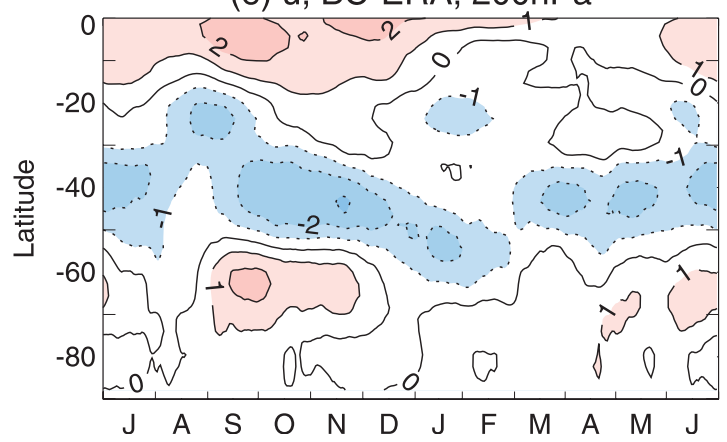

FIG. 3. (a) Zonal-mean zonal wind on the 200-hPa level for each experiment and the reanalysis, averaged over the DJF season; latitude-pressure cross section of the DJF-averaged zonal-mean zonal wind bias for (b) FREE and (c) BC; and zonal-mean zonal wind bias on the 200-hPa level as a function of season smoothed with a 31-day running mean for (d) FREE and (e) BC.

\section{(a) ERA}

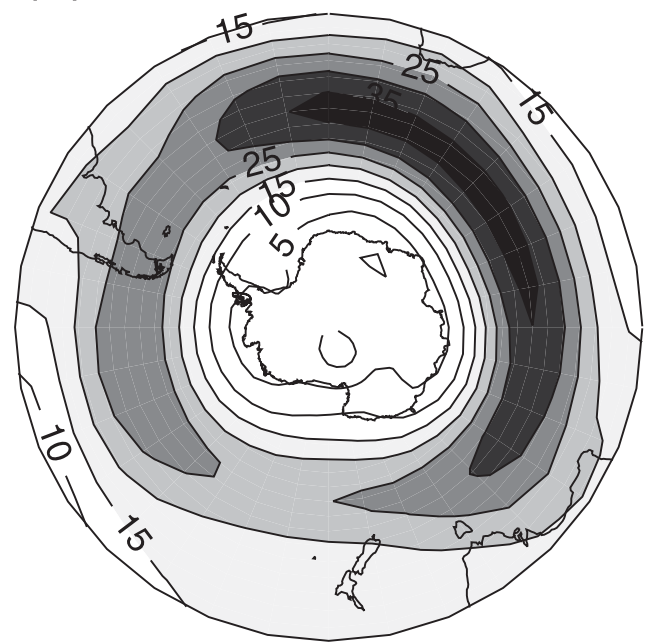

(b) FREE

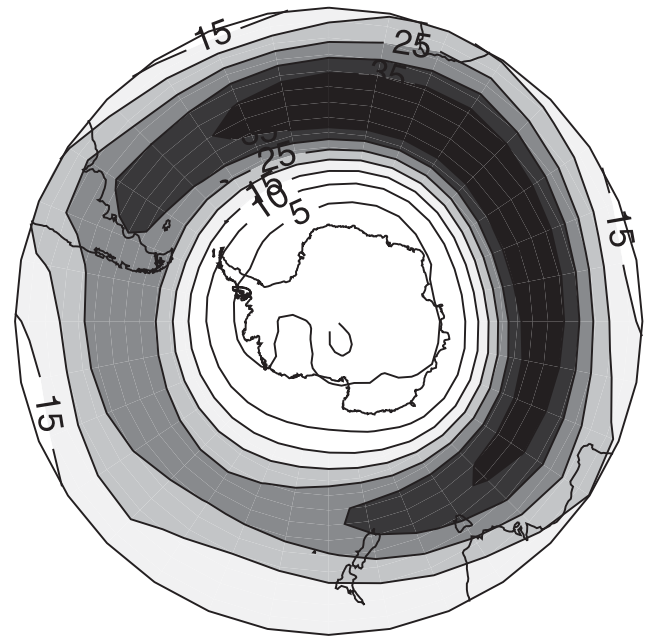

(b) $\mathrm{BC}$

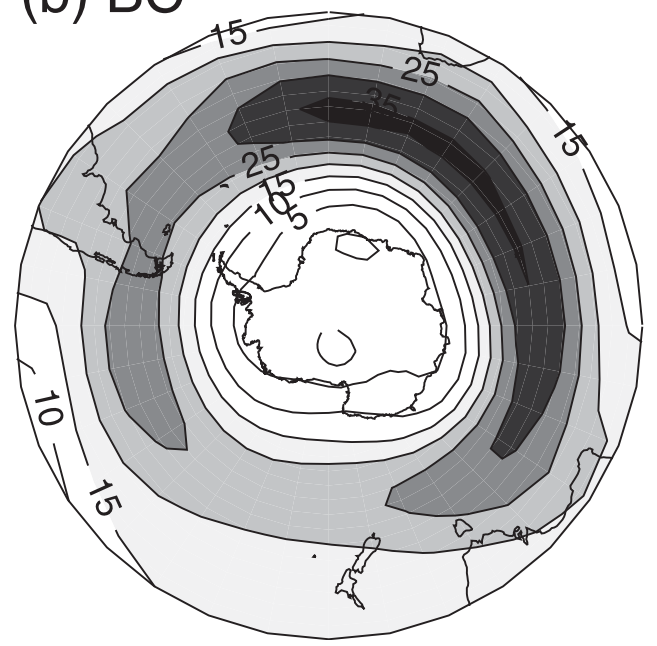

FIG. 4. Climatological zonal wind on the $300-\mathrm{hPa}$ level for the DJF season for (a) ERA, (b) FREE and (c) BC. Contour interval = $5 \mathrm{~m} \mathrm{~s}^{-1}$. 
climatological zonal wind on the $300-\mathrm{hPa}$ level in DJF for ERA, FREE, and BC. The FREE jet is too zonally extended as can be seen by comparison of the longitudinal extent of the 25 and $35 \mathrm{~m} \mathrm{~s}^{-1}$ contours in Figs. $4 \mathrm{a}$, b. There is evidence that jets that are more zonal may exhibit greater persistence (Gerber and Vallis 2007), and it is therefore important that this bias be alleviated if a complete assessment of the influence of climatological zonal wind biases on SAM persistence is to be made. In BC (Fig. 4c), this bias has indeed been alleviated, demonstrating that the full 3D climatology is much closer to that of ERA-Interim when bias correction is applied.

The zonal-mean zonal wind and zonal-mean temperature bias correction tendencies that are used to achieve the zonal-mean improvement are shown in Fig. 5. These are an order of magnitude smaller than the leadingorder terms in the momentum and thermodynamic equations (possibly with the exception of the warming at the Antarctic surface in Fig. 5b). Since these bias correction tendencies are derived from simulations in which the resolved dynamics are being nudged toward those of ERA-Interim, they indicate deficiencies that are associated with unresolved processes. Examination of the bias correction tendencies can therefore indicate where deficiencies in the model lie. For example, the zonal wind tendencies suggest that an improvement is needed in the surface friction since they are acting to decelerate the zonal wind near the surface in regions of westerly wind and accelerate the zonal wind near the surface in regions of easterly wind. The temperature tendencies are trying to warm near the surface over land, suggesting the model is too cold there and among other things, the tendencies are cooling the tropical upper troposphere and warming the high-latitude tropopause region. Which of these features is ultimately the cause of the climatological zonal-mean zonal wind biases remains an open question and will not be discussed further here. Here we are focused on the biases in SAM variability and how they are influenced by these improvements in the climatological circulation under bias correction.

\section{b. The influence on SAM characteristics}

The characteristics of the SAM in each simulation and ERA-Interim are summarized in Fig. 6 using the diagnostics of Gerber et al. (2010). The structure of the SAM at a selection of pressure levels is shown in Fig. 6a. This is obtained, following the method of Gerber et al. (2010), by regressing the zonal-mean geopotential height anomaly $\Phi^{\prime}$ onto the SAM index $P C$ and multiplying by $\cos$ (latitude). Each of the experiments and ERA-Interim are plotted here, but it is difficult to discern the different lines in the troposphere as they are each very similar. For (a) Bias Correction u tendency, DJF

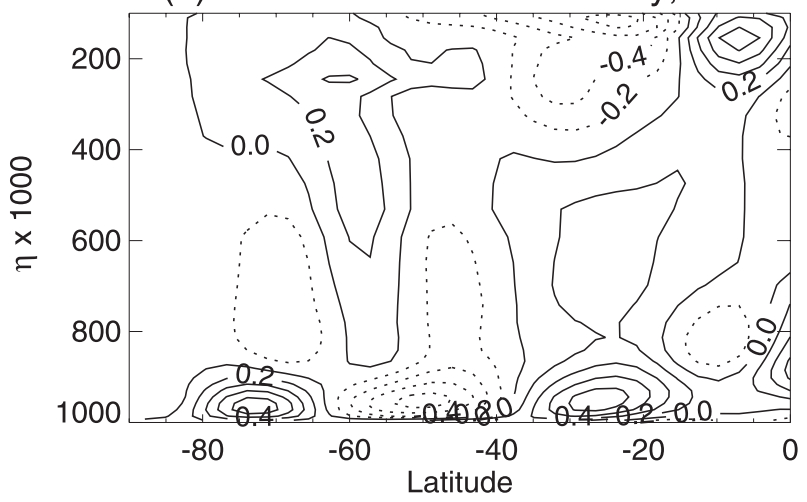

(b) Bias Correction T tendency, DJF

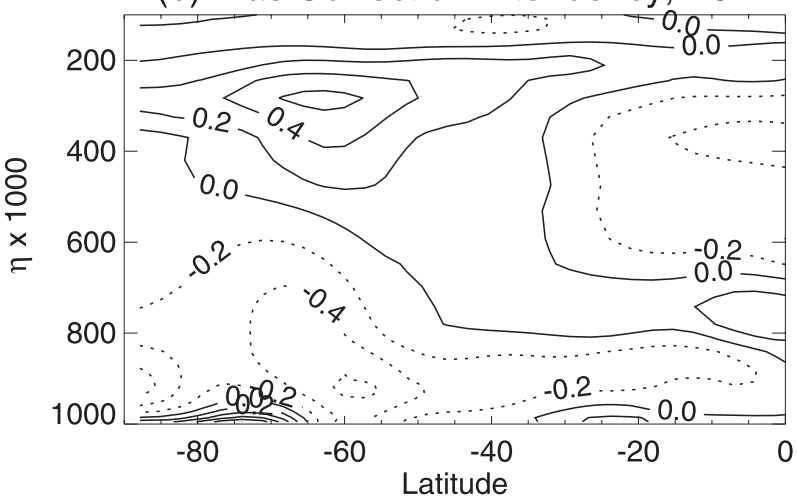

FIG. 5. (a) DJF-averaged (a) zonal-mean zonal wind tendency $\left(\mathrm{m} \mathrm{s}^{-1} \mathrm{day}^{-1}\right.$ ) and (b) zonal-mean temperature tendency $\left(\mathrm{K} \mathrm{day}^{-1}\right)$ applied in the bias correction. Note the vertical axis is CMAM's hybrid sigma/pressure coordinate ( $\eta$ level) multiplied by 1000 , which can be considered an approximate pressure level away from topography.

each experiment and the reanalysis, the tropospheric SAM exhibits a similar latitudinal structure and amplitude, corresponding to geopotential height anomalies that are in geostrophic balance with a latitudinal shift of the jet. At higher altitudes, the effect of the nudging is apparent: the amplitude of SAM variability is extremely damped in NUDG and BCNUDG. It is also clear that, in the CMAM simulations that have stratospheric variability, FREE and BC, the amplitude of the SAM is larger than that in the reanalysis above $30 \mathrm{hPa}$, but this is unlikely to be of concern for investigations of the tropospheric SAM.

Figure $6 \mathrm{~b}$ shows the root-mean-square amplitude of this SAM structure, weighted by $\cos$ (latitude). Some small quantitative differences in the SAM amplitude do actually occur in the troposphere between each of the experiments and the observations, which is not so easily apparent in Fig. 6a. The presence of stratospheric nudging in NUDG and BCNUDG tends to reduce the amplitude of the tropospheric SAM slightly. 
(a) SAM structure

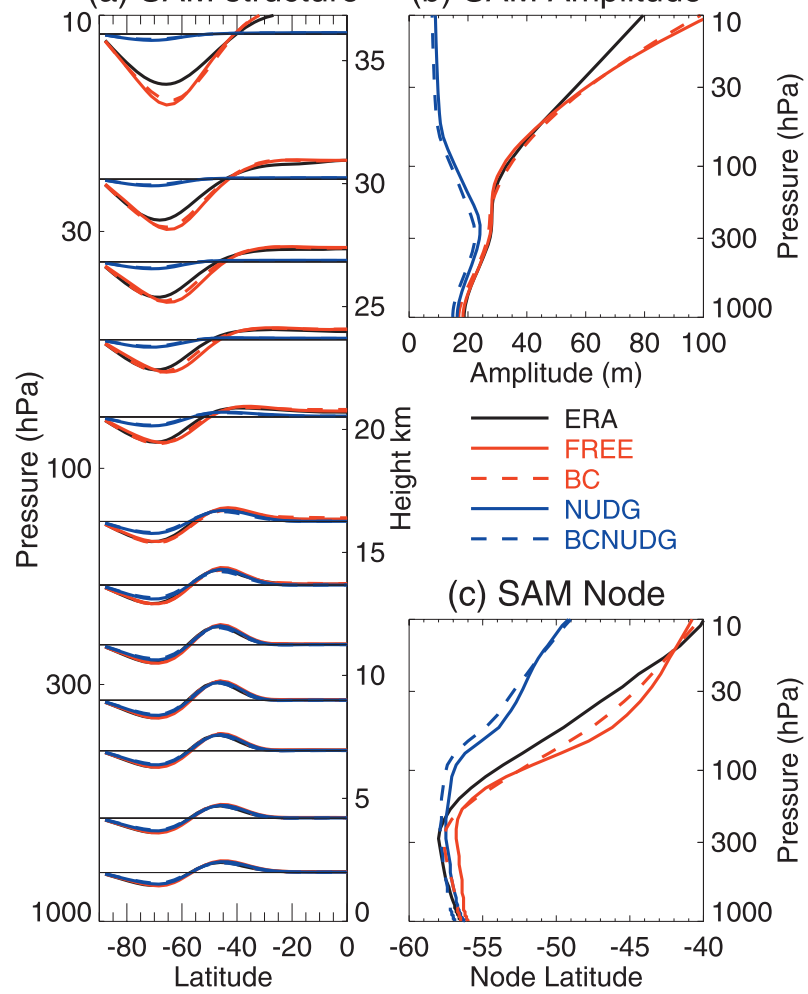

FIG. 6. (a) Latitudinal structure of the SAM obtained by regressing zonal-mean $\Phi^{\prime}$ (in $\mathrm{km}$ ) weighted by $\cos ($ latitude) onto the SAM index, displacing by the height of the relevant pressure level after multiplying by 30 to increase visibility. (b) The root-meansquare amplitude of the SAM weighted by $\cos$ (latitude) as a function of pressure. (c) Latitude of the node of the SAM structure: that is, the zero point of the SAM structure in the midlatitudes. Note that there is a factor of 9.8 difference between the structures in (a) and those in Simpson et al. (2011). Simpson et al. (2011) used geopotential (rather than geopotential height) to calculate the SAM structure and incorrectly stated the units as kilometers.

Finally, the location of the SAM node is shown in Fig. $6 c$. The SAM node is the zero crossing point of the SAM structure in the midlatitudes and was obtained after first interpolating the SAM structure onto a $0.1^{\circ}$ latitude grid using cubic splines. There are only minor differences in the location of the tropospheric node. BC and BCNUDG, which are bias corrected in the troposphere, have their node extremely close to ERA-Interim, as expected. FREE is about $1^{\circ}$ farther equatorward, which is expected given the jet bias. NUDG actually lies closer to ERA-Interim than FREE but, given that the tropospheric climatology is the same in FREE and NUDG (Fig. 3a), this may suggest that this minor difference in SAM node is not actually significant. In the stratosphere, nudging substantially shifts the SAM node poleward. That is because, by damping the variability in the stratosphere, the variability in $\Phi$ at stratospheric levels is

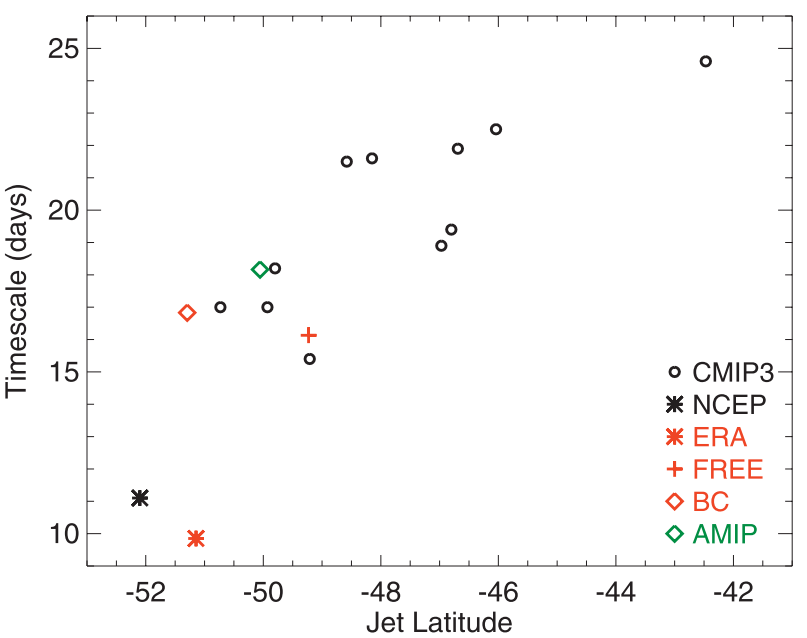

FIG. 7. Annual-mean time scale vs annual-mean jet latitude. The black symbols are from Fig. 1b of KG2010. Circles show each of the individual CMIP3 models analyzed by KG2010, and the black asterisk shows KG2010's values for NCEP reanalysis. The three red symbols represent the data and model simulations analyzed here: ERA-Interim, FREE, and BC. Only FREE and BC are shown, as they do not have a nudged stratosphere and so are more comparable to the reanalyses and the other models in the CMIP3 archive. The green point, labeled AMIP, is an additional free-running simulation with prescribed time-varying SSTs: that is, with the same lower boundary conditions as BC, for comparison.

dominated much more by tropospheric variability, which has its node at a higher latitude.

Overall, the nudging and/or bias correcting has had the desired effect on the SAM. The tropospheric SAM structure and amplitude have been largely unaffected by either methodology, but the bias correction has improved the 3D climatology, and the nudging has severely damped the stratospheric SAM variability.

\section{Results}

The influence of the biases in climatological tropospheric circulation and stratospheric vortex breakdown date on SAM persistence will now be assessed. Before examining the seasonal variation in time scales, the results will first be put in the context of previous research by discussing the annual-mean time scales.

\section{a. Annual-mean time scales}

KG2010 analyzed the annual-mean SAM time scales in 11 of the 23 GCMs from the CMIP3 archive. They found that all the GCMs had SAM time scales that were biased long compared to the reanalysis and that there was a relationship between the extent of this bias and the equatorward bias in the tropospheric zonal-mean climatology. Their results are reproduced in Fig. 7 (black symbols; J. Kidston 2012, personal communication), 
which shows the annual-mean tropospheric SAM time scales versus climatological surface jet latitude for each of the different models and National Centers for Environmental Prediction (NCEP) reanalysis from the KG2010 study. They found a linear relationship: the lower the climatological jet latitude, the greater the SAM persistence. This has led to the hypothesis that alleviating the biases in tropospheric zonal-mean climatology may alleviate the bias in the tropospheric SAM time scales.

We now add the three new points shown in red to the KG2010 plot, calculated in the same way as KG2010, except that the zonal wind on the 995-hPa level, rather than 10-m elevation wind, was used to define the jet latitude. First, ERA-Interim is also included as this is the reanalysis dataset used in the present study. There are only minor differences between this and NCEP. The other two additional red points show the FREE and BC simulations. These two simulations are not nudged in the stratosphere so have full stratospheric zonal-mean variability and are therefore more comparable to the reanalysis and the other CMIP3 simulations. Note that, while $\mathrm{BC}$ is directly comparable to the CMIP3 simulations, FREE is lacking in SST variability and so is not completely comparable. For comparison, an additional free-running simulation with prescribed time-varying SSTs (termed AMIP after the Atmospheric Model Intercomparison Project) is also shown (green point). The time scale is calculated from three ensemble members with prescribed time-varying SSTs from 1970 to 2010. It is found to have an annual-mean time scale that is around 2 days longer than FREE, suggesting that the presence of SST variability enhances SAM persistence slightly and therefore slightly worsens the bias in the free-running model.

The FREE simulation exhibits a climatological equatorward bias in the tropospheric jet of around $2^{\circ}$ latitude. While substantial biases in the zonal wind are evident in Figs. 3a,b, the bias in jet position that this corresponds to is quite small, and this version of CMAM actually appears on the better end of the spectrum of models when the bias is characterized by the location of the near-surface wind maximum. In BC, the troposphere and stratosphere have been bias corrected so the climatology is much improved. The jet location is now very similar to that of the reanalysis, as expected. However, it is clear that there is still a substantial bias in the annualmean SAM time scales, even when these climatological circulation biases (both zonal mean and 3D) have been alleviated.

The jet latitude bias in CMAM is fairly small relative to other models and it may be that improving the jet latitude in models with a more severe jet latitude bias will have a greater impact on SAM time scale. Indeed, a recent study with one such model has demonstrated a substantial improvement in both the jet latitude and SAM persistence by increasing model resolution (Arkelian and Codron 2012). However, this does not rule out the possibility that both the jet latitude bias and the SAM timescale bias have some other underlying common cause since correlations do not establish causality. The aim here is to assess whether the SAM time scale is improved to values close to that of the reanalysis if the model climatological circulation (both jet position and timing of the vortex breakdown) is improved. For CMAM, the answer is no and this is particularly true in the summer season as will be discussed below. It is possible that this is also the case for other models since, if a straight line were fit through the model points in Fig. 7, it would predict a significantly more persistent SAM than found in the reanalysis for realistic jet latitudes, suggesting that improving the jet latitude alone would not solve the problem. So, overall this suggests that, aside from the tropospheric jet latitude bias, there may be another underlying general cause of the enhanced SAM persistence in models, relative to observations.

\section{b. The seasonal variation in time scales}

The SAM time-scale bias exhibits a pronounced seasonal variation (Gerber et al. 2008a, 2010), so we now proceed to examine the seasonal variation in SAM time scales in each of the four CMAM simulations and ERAInterim. The SAM time scales as a function of season and height are shown in Fig. 8. ERA-Interim (Fig. 8a), FREE (Fig. 8b), and NUDG (Fig. 8c) have already been discussed in Simpson et al. (2011). In ERA-Interim, the tropospheric SAM time scales maximize in November and December, around the timing of the vortex breakdown when stratospheric variability is important in contributing to enhanced SAM persistence. FREE shows a similar increase in persistence starting from October, but the SAM time scales become much longer than those in the reanalysis and the enhanced persistence extends much later in the summer season. As a result, the time scales are considerably biased in the model for the whole of the summer season, in a similar manner to most other GCMs (Gerber et al. 2008a, 2010).

The NUDG simulation (Fig. 8c) was used in Simpson et al. (2011) to demonstrate that, even in the absence of stratospheric variability, the tropospheric SAM time scales in CMAM exhibit a pronounced seasonal variation, with enhanced time scales in the summer season that are biased long compared to the reanalysis. This led Simpson et al. (2011) to conclude that there must be a contribution to the summertime SAM time-scale bias from internal tropospheric dynamics. 

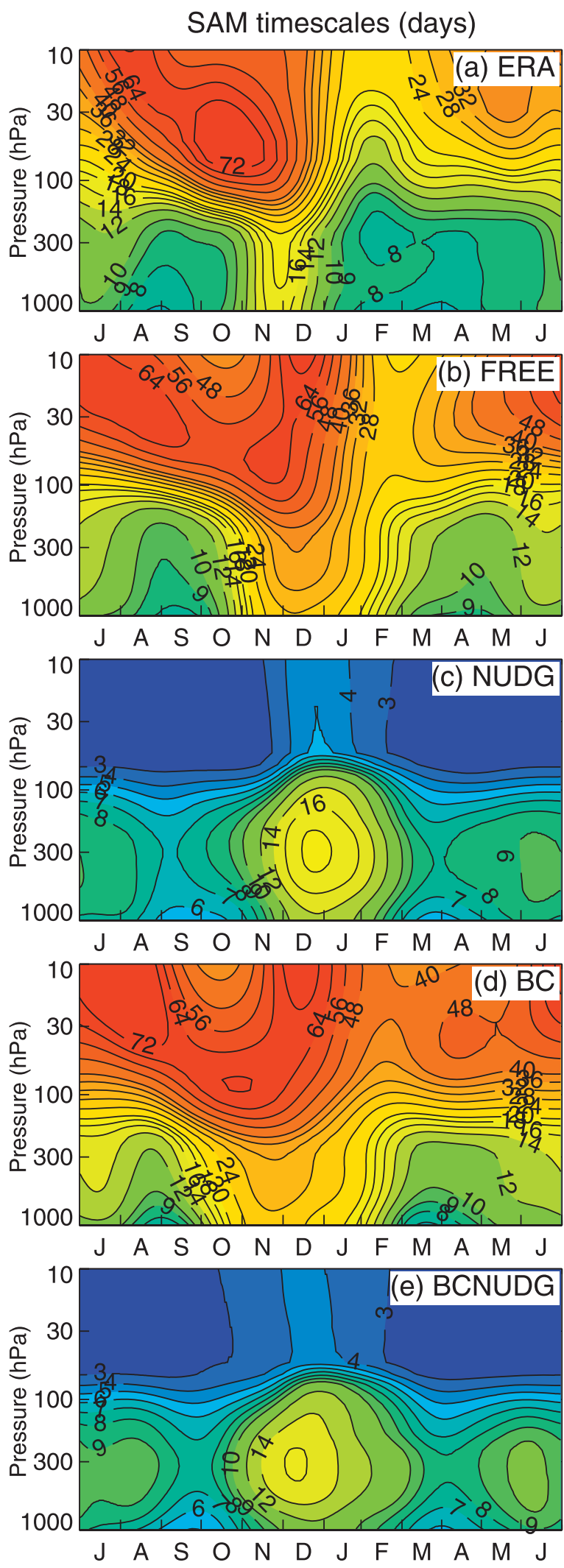

FIG. 8. SAM time scale (in days) as a function of month and pressure for (a) ERA, (b) FREE, (c) NUDG, (d) BC, and (e) BCNUDG.

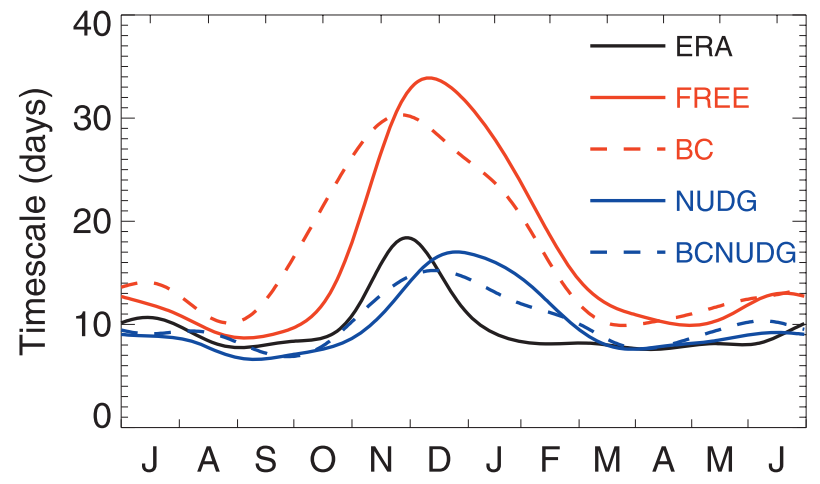

FIG. 9. SAM time scale (in days) as a function of month on the 531-hPa level for each experiment and ERA-Interim.

Figure $8 \mathrm{~d}$ shows the results for $\mathrm{BC}$, where stratospheric variability is present, but both the troposphere and stratosphere have been bias corrected to be close to the reanalysis climatology. In the DJF season, the climatological jet position in ERA-Interim lies around $50^{\circ} \mathrm{S}$ whereas FREE is biased equatorward by about $2^{\circ}$. Therefore, the magnitude of the jet latitude bias is similar to that seen in the annual mean in Fig. 7 and the bias correction virtually alleviates this, as seen in Figs. 3b,c.

With the bias correction, the peak in tropospheric time scales has been shifted slightly earlier in the season (cf. Fig. 8b). This is also true when comparing BC with the AMIP simulation (not shown), so it is probable that this shift in the timing of the tropospheric maximum is coming from the shift to an earlier breakdown date of the stratospheric vortex, resulting from the bias correction. However, in spite of these improvements in the timing of the stratospheric variability maximum, it is clear that the bias in tropospheric summertime SAM time scales remains. The simulations and reanalysis can be compared more directly in Fig. 9, where the time scales near the $500-\mathrm{hPa}$ level are plotted. The timing of the peak in tropospheric SAM time scales in BC is now quite similar to the reanalysis, occurring in late November/early December. While there is a slight improvement in time scales over the summer season, they are still approximately double those of the reanalysis.

In NUDG and BCNUDG, stratospheric variability is removed by nudging and climatological SSTs are prescribed. Therefore, these experiments can tell us about biases that are related to dynamics that may be considered more internal to the dynamics of variability of the tropospheric jet. The SAM persistence of BCNUDG (Fig. 8e) demonstrates that it still exhibits a seasonal variation in SAM time scales in the absence of stratospheric variability, with the time scales maximizing in 
the summer. The time scales are slightly reduced compared to NUDG in the summer season but enhanced in the winter. It is clear, however, that, even in the absence of stratospheric zonal-mean and SST variability, the model has longer tropospheric time scales in the summer than does the reanalysis. This is clear by a direct comparison of the experiments in Fig. 9. Since stratospheric zonal-mean and SST variability are only likely to increase the persistence of the SAM, BCNUDG demonstrates that a bias in the internal tropospheric dynamics of the SAM, not caused by biases in the climatological circulation, remains.

\section{Discussion and conclusions}

Previous research has identified a major deficiency in the ability of comprehensive GCMs to simulate the $\mathrm{SAM}$ in the $\mathrm{SH}$ summer season in the troposphere (Gerber et al. 2008a, 2010). Most models, whether they are coupled to an ocean or not, exhibit SAM variability that is much too persistent in the summer months. There are various different factors that could contribute to this, two of which have been examined here. One factor is the common problem that the Antarctic vortex tends to break down too late (Butchart et al. 2011); the other is that the climatological tropospheric midlatitude jet tends to be too far equatorward in models (Fyfe and Saenko 2006; KG2010; Swart and Fyfe 2012a,b).

Here we have presented simulations involving nudging and bias correcting that have allowed us to alleviate both these biases, albeit in an artificial way, to examine their impact on SAM time scales. It is found, in the BC experiment, that improving both the timing of the vortex breakdown and the climatological tropospheric jet structure does not substantially improve the SAM time scales. While the improvements in the timing of the vortex breakdown do improve the seasonal timing of the maximum in tropospheric persistence, the SAM is still much too persistent throughout the summer season. Furthermore, the BCNUDG experiment has revealed that even in the absence of stratospheric zonal-mean and SST variability and, with an improved climatological tropospheric jet structure, the SAM time scales in the troposphere are biased long compared to the reanalysis (in which stratospheric variability provides an additional enhancement of the time scales). Simpson et al. (2011) had already identified a role for internal tropospheric dynamics in contributing to the summertime SAM timescale bias, and here it is found that this bias in internal tropospheric dynamics remains even when the climatological jet structure is improved. This suggests that, while the influence of climatological biases in the zonal wind on eddy propagation and feedbacks may be contributing to some of the bias in SAM persistence (particularly in models with a more severe jet bias), another factor is contributing significantly to the bias, particularly in the summer months. We can conclude with certainty that this is the case for CMAM.

Whether the internal tropospheric bias is the whole story remains to be seen. Comparing FREE with NUDG or BC with BCNUDG demonstrates that stratospheric variability plays a significant role in enhancing the SAM time scales, as already discussed in Simpson et al. (2011). However, whether it would have as large an influence if there was no bias in internal tropospheric dynamics is unclear. In particular, if the persistence bias in the simulations without stratospheric variability is due to tropospheric eddy feedbacks that are too strong, then it is entirely possible that this same bias in eddy feedbacks could cause the stratosphere to have an unrealistically strong influence on the tropospheric SAM. Determining whether this is the case is beyond the scope of this study and most likely will require the bias in internal tropospheric dynamics to be understood and alleviated. Progress could then be made in understanding other possible contributing factors to biases in SAM persistence. What can be concluded from the experiments performed is that a bias in internal tropospheric dynamics, but not caused by the tropospheric jet structure bias, plays a role in the tropospheric summertime SAM time-scale bias in CMAM and in Part II we proceed to investigate the possible causes of this internal tropospheric bias.

An important point is that here we have artificially improved the climatological jet structure by bias correcting. We can conclude that the tropospheric jet structure bias is not causing the SAM time-scale bias in CMAM, but it is possible that both these problems have a common cause. In Part II, we find that a dominant contribution to the SAM persistence bias is coming from a deficiency in planetary wave feedbacks on the SAM, which may be related to an under representation of planetary-scale waves in the SH. It is possible that the climatological momentum fluxes (or rather the lack thereof) associated with such waves are contributing to the climatological zonal wind biases, but Fig. 5 also reveals deficiencies in parameterized processes such as surface friction or diabatic heating in the tropics. Which of these features are the most important for improving the climatological jet position in models and to what extent the jet latitude bias and SAM persistence bias have a common cause remain open questions. As far as the SAM time-scale bias is concerned, it is important to find the underlying cause that exists even when the jet structure is close to the reanalysis, and it is this problem that is addressed in Part II. 
Acknowledgments. This work was funded by the Natural Sciences and Engineering Research Council of Canada and Environment Canada. Computing resources were provided by Environment Canada. We are grateful to Joe Kidston for providing the data from the KG2010 study used in Fig. 7. We are also grateful to Michael Sigmond for making his data for the BC simulation available for this study and to Slava Kharin for providing technical assistance in the setup of the bias correction. We are also grateful to Charles McLandress, John Fyfe, and four anonymous reviewers for helpful comments on this manuscript.

\section{REFERENCES}

Arkelian, A., and F. Codron, 2012: Southern Hemisphere jet variability in the IPSL GCM at varying resolutions. J. Atmos. Sci., 69, 3788-3799.

Baldwin, M. P., and D. W. J. Thompson, 2009: A critical comparison of stratosphere-troposphere coupling indices. Quart. J. Roy. Meteor. Soc., 135, 1661-1672.

- D. B. Stephenson, D. W. J. Thompson, T. J. Dunkerton, A. J. Charlton, and A. O'Neill, 2003: Stratospheric memory and skill of extended-range weather forecasts. Science, 301, 636-640, doi:10.1126/science.1087143.

,-- , and I. T. Jollife, 2009: Spatial weighting and iterative projection methods for EOFs. J. Climate, 22, 234-243.

Barnes, E. A., and D. L. Hartmann, 2010: Dynamical feedbacks of the southern annular mode in winter and summer. J. Atmos. Sci., 67, 2320-2330.

,-- D. M. W. Frierson, and J. Kidston, 2010: Effect of latitude on the persistence of eddy-driven jets. Geophys. Res. Lett., 37, L11804, doi:10.1029/2010GL043199.

Butchart, N., and Coauthors, 2011: Multimodel climate and variability of the stratosphere. J. Geophys. Res., 116, D05102, doi:10.1029/2010JD014995.

Dee, D. P., and Coauthors, 2011: The ERA-Interim reanalysis: Configuration and performance of the data assimilation system. Quart. J. Roy. Meteor. Soc., 137, 553-597, doi:10.1002/ qj.828.

Fogt, R. L., J. Perlwitz, A. J. Monaghan, D. H. Bromwich, J. M. Jones, and G. J. Marshall, 2009: Historical SAM variability. Part II: Twentieth-century variability and trends from reconstructions, observations, and the IPCC AR4 models. J. Climate, 22, 5346-5365.

Fyfe, J. C., and O. A. Saenko, 2006: Simulated changes in the extratropical Southern Hemisphere winds and currents. Geophys. Res. Lett., 33, L06701, doi:10.1029/2005GL025332.

Gerber, E. P., and G. K. Vallis, 2007: Eddy-zonal flow interactions and the persistence of the zonal index. J. Atmos. Sci., 64, 32963311.

—, L. M. Polvani, and D. Ancukiewicz, 2008a: Annular mode time scales in the Intergovernmental Panel on Climate Change Fourth Assessment Report models. Geophys. Res. Lett., 35, L22707, doi:10.1029/2008GL035712.

—, S. Voronin, and L. M. Polvani, 2008b: Testing the annular mode autocorrelation time scale in simple atmospheric general circulation models. Mon. Wea. Rev., 136, 1523-1536.

_ , and Coauthors, 2010: Stratosphere-troposphere coupling and annular mode variability in chemistry-climate models. J. Geophys. Res., 115, D00M06, doi:10.1029/2009JD013770.
Gong, D., and S. Wang, 1999: Definition of Antarctic Oscillation index. Geophys. Res. Lett., 26, 459-462.

Haigh, J. D., M. Blackburn, and R. Day, 2005: The response of tropospheric circulation to perturbations in lower-stratospheric temperature. J. Climate, 18, 3672-3685.

Keeley, S. P. E., R. T. Sutton, and L. C. Shaffrey, 2009: Does the North Atlantic Oscillation show unusual persistence on intraseasonal timescales? Geophys. Res. Lett., 36, L22706, doi:10.1029/2009GL040367.

Kharin, V. V., and J. F. Scinocca, 2012: The impact of model fidelity on seasonal predictive skill. Geophys. Res. Lett., 39, L18803, doi:10.1029/2012GL052815.

Kidston, J., and E. P. Gerber, 2010: Intermodel variability of the poleward shift of the austral jet stream in the CMIP3 integrations linked to biases in 20th century climatology. Geophys. Res. Lett., 37, L09708, doi:10.1029/ 2010GL042873.

—, D. M. W. Frierson, J. A. Renwick, and G. K. Vallis, 2010: Observations, simulations, and dynamics of jet stream variability and annular modes. J. Climate, 23, 6186-6199.

Leith, C. E., 1975: Climate response and fluctuation dissipation. J. Atmos. Sci., 32, 2022-2026.

Lorenz, D. J., and D. L. Hartmann, 2001: Eddy zonal flow feedback in the Southern Hemisphere. J. Atmos. Sci., 58, 3312-3327.

— and - 2003: Eddy-zonal flow feedback in the Northern Hemisphere winter. J. Climate, 16, 1212-1227.

— wind response to global warming in the IPCC scenario integrations. J. Geophys. Res., 112, D10119, doi:10.1029/ 2006JD008087.

McLandress, C., T. G. Shepherd, J. F. Scinocca, D. A. Plummer, M. Sigmond, A. I. Jonsson, and M. C. Reader, 2011: Separating the dynamical effects of climate change and ozone depletion. Part II: Southern Hemisphere troposphere. J. Climate, 24, 1850-1868.

Miller, R. L., G. A. Schmidt, and D. T. Shindell, 2006: Forced annular variations in the 20th century Intergovernmental Panel on Climate Change Fourth Assessment Report models. J. Geophys. Res., 111, D18101, doi:10.1029/2005JD006323.

Polvani, L. M., M. Previdi, and C. Deser, 2011: Large cancellation, due to ozone recovery, of future Southern Hemisphere atmospheric circulation trends. Geophys. Res. Lett., 38, L04707, doi:10.1029/2011GL046712.

Rayner, N. A., D. E. Parker, E. B. Horton, C. K. Folland, L. V. Alexander, D. P. Rowell, E. C. Kent, and A. Kaplan, 2003: Global analyses of sea surface temperature, sea ice, and night marine air temperature since the late nineteenth century. J. Geophys. Res., 108, 4407, doi:10.1029/2002JD002670.

Robinson, W. A., 1996: Does eddy feedback sustain variability in the zonal index? J. Atmos. Sci., 53, 3556-3569.

_ 2000: A baroclinic mechanism for the eddy feedback on the zonal index. J. Atmos. Sci., 57, 415-422.

Russell, J. L., K. W. Dixon, A. Gnanadesikan, R. J. Stouffer, and J. R. Toggweiler, 2006: The Southern Hemisphere westerlies in a warming world: Propping open the door to the deep ocean. J. Climate, 19, 6382-6390.

Scinocca, J. F., N. A. McFarlane, M. Lazare, J. Li, and D. Plummer, 2008: Technical note: The CCCma third generation AGCM and its extension into the middle atmosphere. Atmos. Chem. Phys., 8, 7055-7074.

Seager, R., N. Harnik, Y. Kushnir, W. Robinson, and J. Miller, 2003: Mechanisms of hemispherically symmetric climate variability. J. Climate, 16, 2960-2978. 
Sigmond, M., and J. C. Fyfe, 2010: Has the ozone hole contributed to increased Antarctic sea ice extent? Geophys. Res. Lett., 37, L18502, doi:10.1029/2010GL044301.

Simpson, I. R., M. Blackburn, J. D. Haigh, and S. N. Sparrow, 2010: The impact of the state of the troposphere on the response to stratospheric heating in a simplified GCM. J. Climate, 23, 6166-6185.

_, P. Hitchcock, T. G. Shepherd, and J. F. Scinocca, 2011: Stratospheric variability and tropospheric annular-mode timescales. Geophys. Res. Lett., 38, L20806, doi:10.1029/ 2011 GL049304.

_- M. Blackburn, and J. D. Haigh, 2012: A mechanism for the effect of tropospheric jet structure on the annular mode-like response to stratospheric forcing. J. Atmos. Sci., 69, 21522170.

, T. G. Shepherd, P. Hitchcock, and J. F. Scinocca, 2013: Southern annular mode dynamics in observations and models. Part II: Eddy feedbacks. J. Climate, in press.

Son, S.-W., and S. Lee, 2005: The response of westerly jets to thermal driving in a primitive equation model. J. Atmos. Sci., 62, 3741-3757.
S. B. Feldstein, and J. E. Ten Hoeve, 2008: Time scale and feedback of zonal-mean-flow variability. J. Atmos. Sci., 65, 935-952.

- and Coauthors, 2010: The impact of stratospheric ozone on the Southern Hemisphere circulation changes: A multimodel assessment. J. Geophys. Res., 115, D00M07, doi:10.1029/ 2010JD014271.

Swart, N. C., and J. C. Fyfe, 2012a: Observed and simulated changes in Southern Hemisphere surface westerlies. Geophys. Res. Lett., 39, L16711, doi:10.1029/2012GL052810.

$\longrightarrow$, and $\longrightarrow, 2012 \mathrm{~b}$ : Ocean carbon uptake and storage influenced by wind bias in global climate models. Nat. Climate Change, 2, 47-52.

Thompson, D. W. J., and J. M. Wallace, 2000: Annular modes in the extratropical circulation. Part I: Month-to-month variability. J. Climate, 13, 1000-1016.

and S. Solomon, 2002: Interpretation of recent Southern Hemisphere climate change. Science, 296, 895-899, doi:10.1126/science.1069270.

Uppala, S. M., and Coauthors, 2005: The ERA-40 Re-Analysis. Quart. J. Roy. Meteor. Soc., 131, 2961-3012. 